\title{
Using Event Representations to Generate Robot Semantics
}

\author{
PETER GÄRDENFORS, Lund University and University of Technology Sydney
}

\begin{abstract}
Most semantic models employed in human-robot interactions concern how a robot can understand commands, but in this article the aim is to present a framework that allows dialogic interaction. The key idea is to use events as the fundamental structures for the semantic representations of a robot. Events are modeled in terms of conceptual spaces and mappings between spaces. It is shown how the semantics of major word classes can be described with the aid of conceptual spaces in a way that is amenable to computer implementations. An event is represented by two vectors, one force vector representing an action and one result vector representing the effect of the action. The two-vector model is then extended by the thematic roles so an event is built up from an agent, an action, a patient, and a result. It is shown how the components of an event can be put together to semantic structures that represent the meanings of sentences. It is argued that a semantic framework based on events can generate a general representational framework for humanrobot communication. An implementation of the framework involving communication with an iCub will be described.
\end{abstract}

CCS Concepts: • Computing methodologies $\rightarrow$ Natural language generation; Lexical semantics; $\mathbf{C o g}$ nitive robotics; Cognitive science; $\bullet$ Software and its engineering $\rightarrow$ Semantics;

Additional Key Words and Phrases: Semantic models, human-robot communication, conceptual spaces, event cognition, word classes, sentence generation

\section{ACM Reference format:}

Peter Gärdenfors. 2019. Using Event Representations to Generate Robot Semantics. ACM Trans. Hum.-Robot Interact. 8, 4, Article 21 (October 2019), 21 pages.

https://doi.org/10.1145/3341167

\section{INTRODUCTION}

The problem in focus of this article is how a robot can learn semantic representations that are appropriate for human-robot communication (HRC). Earlier attempts to solve this problem in AI and robotics have focused on defining logical ontologies, symbol manipulation, and following syntactic rules. Such logical approaches to language generation for robots have, however, not produced results that are general and flexible enough to function in real-world robot applications.

To be sure, learning to use a language involves learning its words-its lexicon; and how to put the words together in an appropriate way-its syntax. More importantly, however, the meaning of the words must be learned and what they mean when combined into sentences. If you do not master the semantics of the language you are using, then there is no point in knowing its syntax (lest you are a parrot). Therefore, as regards communication, including that between robots and humans, semantic knowledge is more fundamental than syntactic. Taniguchi et al. [1] present a

Authors' address: P. Gärdenfors, Cognitive Science, Department of Philosophy, LUX, Lund University, Box 192, S-221 00 Lund, Sweden; email: Peter.Gardenfors@lucs.lu.se.

Permission to make digital or hard copies of all or part of this work for personal or classroom use is granted without fee provided that copies are not made or distributed for profit or commercial advantage and that copies bear this notice and the full citation on the first page. Copyrights for components of this work owned by others than the author(s) must be honored. Abstracting with credit is permitted. To copy otherwise, or republish, to post on servers or to redistribute to lists, requires prior specific permission and/or a fee. Request permissions from permissions@acm.org.

(C) 2019 Copyright held by the owner/author(s). Publication rights licensed to ACM.

2573-9522/2019/10-ART21

https://doi.org/10.1145/3341167 
review of different approaches to how the meaning of words in robotics can be acquired and how the meanings can be grounded in perceptual processes. Their focus is, however, on how single words are learned. Within research on HRC, the focus has been on how robots can be made to understand commands, but here my aim is to present a framework that allows for a more dialogic or conversational form.

The new approach proposed here is to let events form the fundamental structures for the semantic representations of a robot. Event cognition is currently much investigated in the cognitive sciences (see, e.g., References [2-4]) and events are considered to be central in human thinking. Therefore, it is natural to let similar structures form the basis for the semantic representations of a robot that communicates with humans. My goal is to present an implementable semantic framework that can be used both for production and understanding of language in HRC.

I model events with the aid of a geometric approach $[2,5]$, representing event structures in terms of vector spaces and mappings between such spaces. The core idea is that an event consists of two vectors, one force vector representing an action and one result vector representing the effect of the action. The two-vector model is then extended with the "thematic roles" in semantics [6], where an event is built up from an agent, an action, a patient, a result, and possibly other roles such as instrument, recipient, and beneficiary. Agent, patient, and other thematic roles are realized by objects (animate or inanimate) that have various properties.

After introducing conceptual spaces as a modeling framework and presenting the event model in greater detail, the main part of the article is devoted to giving a description of the semantics of the components of the event model. In the final part I turn to how the components are put together to semantic structures that represent the meanings of sentences. The basic idea is that a sentence expresses an event $[5,7]$. The event model is introduced in a semi-computational manner, and an implementation for an iCub robot is presented in Section 5.4 compared to other approaches in Section 5.5.

\section{CONCEPTUAL SPACES AS A REPRESENTATIONAL FRAMEWORK}

Within HRC, most models have been based either on symbolic structures focusing on grammar or neural networks that have been trained on various forms of data. A central problem for the symbolic approach in relation to HRC is the grounding problem [8]; that is, attaching meanings to the symbols, while the main problems for the neural network is their slow learning and the opaqueness of what they learn. I have argued that an intermediate form of meaning representations based on geometrical structures in conceptual spaces can overcome these problems [9]. The semantic framework presented here is an extension of the theory of conceptual spaces.

\subsection{The Basics of Conceptual Spaces}

My aim in this section is to present conceptual spaces as a general modeling tool for the components of the event model $[5,9]$. A conceptual space consists of a number of quality dimensions. Examples of quality dimensions are size, temperature, weight, brightness, pitch, and force, as well as the three ordinary spatial dimensions of height, width, and depth. Some quality dimensions are of an abstract non-sensory character; for example, kinship dimensions. An object will be represented by assigning a value to each of the dimensions that characterize the object. Distances between such representations of objects then measure how similar the objects are to each other, where the similarity is not overall similarity but similarity in the respect-color, taste, shape, and so on-that the space is aimed to model.

Conceptual spaces are, as theoretical constructions, mathematical entities, specifically, oneor multi-dimensional structures with a metric defined on them. If the domains have a metric, then one can talk about distances in the conceptual space. Such distances represent degrees of 
similarity between the objects represented in the space so the closer two objects are in the space, the more similar they are. It is assumed that each of the quality dimensions is endowed with certain topological or geometric structures. As an example, take the dimension of time. In science, time is a one-dimensional structure that is isomorphic to the line of real numbers. If now is seen as the zero point on the line, then the future corresponds to the infinite positive real line and the past to the infinite negative line.

The quality dimensions are grouped into domains. For example, the space domain consists of the dimensions width, depth, and height, and the color domain of the dimensions hue, saturation, and brightness. The primary function of the domains is to represent various qualities of objects. The domains are described with the aid of different topological or metric structures. For example, the ordinary space domain forms a three-dimensional Euclidean space, the color domain forms a double cone [9], and the domain of musical harmony forms a torus [10].

A computational implementation of a domain involves specifying its geometric structure together with a distance measure that represents similarities [5, 11]. In many cases this can be done using a standard Cartesian coordinate system and a Euclidean metric. However, for some domains-for example, the color circle-polar coordinates and polar distances are better suited. As we shall see, polar coordinates are also appropriate for the semantics of prepositions.

\subsection{Properties}

The general description of conceptual spaces will now be used to introduce a distinction between properties and object categories. These two types are the most fundamental classes of concepts, but there are also other types. I use the notion of a property to denote information related to a single domain. The following criterion has been proposed $[5,9,12]$, where the geometrical characteristics of the quality dimensions are used to introduce a spatial structure to properties:

Criterion P: A property is a convex region in a single domain.

For example, the property red is a convex region of the color domain and the property hot is a convex region of the temperature domain. ${ }^{1}$

The motivation for the thesis is that, if some objects represented at points $x$ and $y$ in relation to some domain are both examples of a property, then any object that is located between $x$ and $y$ with respect to the same domain will also be an example of the property. Even though not all domains in a conceptual space may have a metric, I assume that the notion of betweenness is defined for all domains. ${ }^{2}$ This will make it possible to apply the criterion for properties generally.

Convexity may seem a strong assumption, but it is a remarkably regular feature of many perceptually grounded domains; for example, color [13], vowels [14], and actions [15]. Although a main argument for convexity is that it facilitates the learnability and generalizability of concepts $[9,16]$, it is also crucial for assuring the effectiveness of communication [17].

There are interesting comparisons to make between analyzing properties (and other concepts) as convex regions and the prototype theory developed by Rosch and her collaborators [18, 19]. Given a convex region, one can describe positions in that region as being more or less central and define prototypes to be the most central points.

Conversely, assume that some conceptual space is given, and that it should be decomposed into a number of regions. If one starts from a set of prototypes, then these can be taken as the central points for the concepts they represent. If the space has a metric, then the information about prototypes can then be used to generate regions by stipulating that any point within the space

\footnotetext{
${ }^{1}$ The borders of the regions need not be sharp. Vague concepts can also be modeled [21].

${ }^{2}$ Convexity can be defined for graphs and orderings, among other structures.
} 


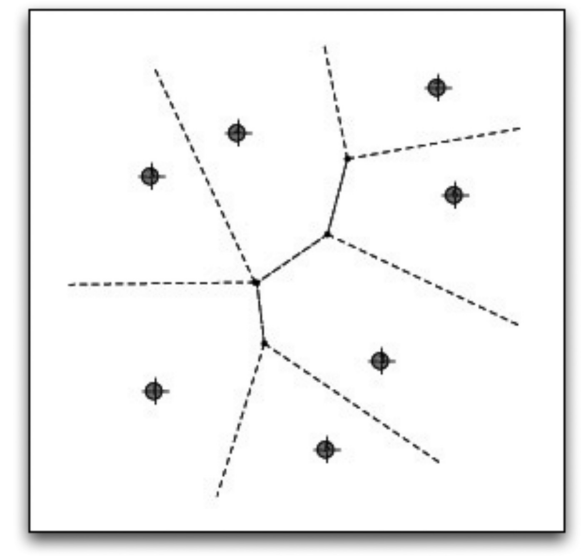

Fig. 1. A Voronoi tessellation of a 2D space into convex sets. The dots represent prototypes.

belongs to the same concepts as the closest prototype. This rule will generate a so-called Voronoi tessellation (see Figure 1). An important feature of Voronoi tessellations is that they always generate a convex partitioning of the space $[9,20]$. It should be noted, however, that for many (typically one-dimensional) domains, prototypes cannot be found. For example, along the temperature dimension, there is no prototype for hot.

Using Voronoi tessellations is a computationally very efficient way of representing concepts. Once the prototypical points for the concepts have been identified and a metric is given, then the tessellation can easily be calculated.

\subsection{Learning New Concepts}

It would be a mistake to provide a robot with all the concepts it may use in problem solving, planning, and communication. A flexible robot must be able to learn many of the concepts it needs from its interaction with the world and with humans. In other words, a robot should be provided with an efficient mechanism for learning new concepts. Learning a concept often proceeds by generalizing from a limited number of exemplars of the concept (see, for example, References [2225]). Adopting the idea that concepts have prototypes, one can assume that a typical instance of the concept is extracted from these exemplars. If the exemplars are described as points in a conceptual space, then a simple rule that can be employed for calculating the prototype from a class of exemplars is that the prototype is defined to be the mean of the positions for all the exemplars [24]. These prototypes can then be used to generate a Voronoi tessellation. Applying this rule means that a prototype is not assumed to be given a priori, but it is instead determined by the perceptual experience of the robot. Figure 2 shows how a set of nine exemplars (represented as differently filled circles), grouped into three categories, generates three prototypical points by calculating means (represented as black crosses) in the space. These prototypes then determine a Voronoi tessellation of the space.

The mechanism illustrated here shows how, once the structure of a domain is established, concepts can be generated from only a few examples. The additional information that is required for the generalization is extracted from the geometrical structure of the underlying conceptual space. In this way, conceptual spaces add information to what is given by the perceptual input.

Furthermore, the concepts generated by such a categorization mechanism are dynamic in the sense that when the robot observes a new item in a category, the prototype for that category will, in general, change somewhat, since the mean of the class of examples will normally change. 


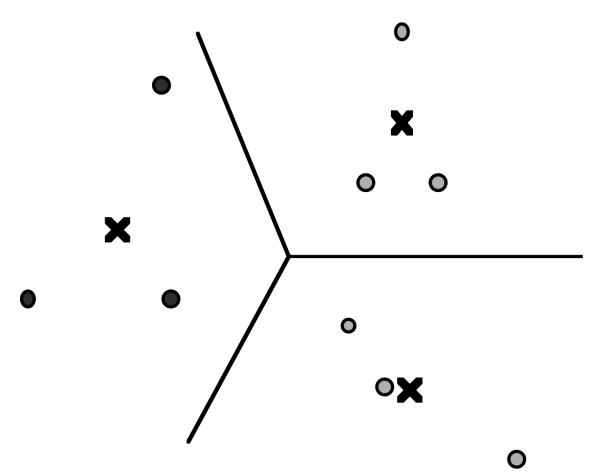

Fig. 2. Voronoi tessellation generated by three classes of exemplars. The crosses represent the prototypes.

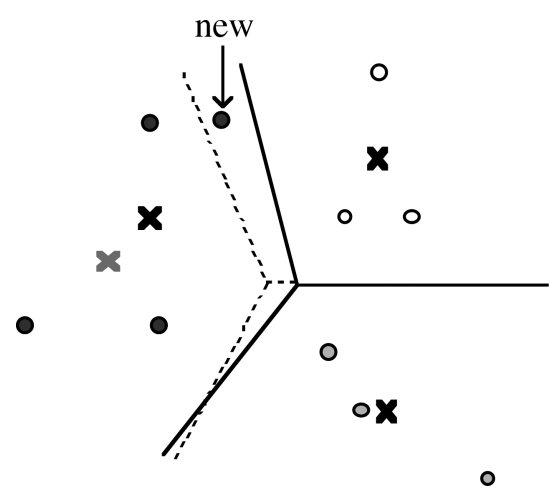

Fig. 3. Change of Voronoi tessellation after learning a new exemplar. The old tessellation is marked by hatched lines, and the old prototype is marked by a gray cross.

Figure 3 shows how the partitioning in Figure 2 is changed after learning about one new exemplar, marked by an arrow, belonging to one of the categories. This addition shifts the prototype of that category, which is defined as the mean of the exemplars, and consequently the Voronoi tessellation is changed. The old tessellation is marked by hatched lines and the old prototype is marked by a gray cross. Categories bordering on the changed prototype will then also change their meaning.

It should be noted that, in contrast to the learning mechanisms of neural networks, the learning mechanism outlined here leads to very quick learning. Given only one or a couple of examples of a category, the robot can calculate an approximate prototype. If a new example of a category is observed, then the previous number of observations of a category will determine how much the prototype should be adjusted. The larger the number of previous observations, the smaller the effect of a new observation will become. The mechanism thus explains how a robot can quickly pick up an approximation of the meaning of a word, but a more precise mastery of the meaning requires several perceived exemplars.

A more general question is how the structure of a conceptual space itself can be acquired by a robot. A brute method is that the programmer provides the structure, in terms of vector representations, so the perceptual input of the robot is transformed into the given structure. Another more biologically valid approach is to let the robot itself learn the relevant underlying spatial structure. An interesting example of such a methodology is presented by Steels and Belpaeme [26], who show that a color space and its partitioning into color concepts can be learned by a combination of sensory input and linguistic exchanges. Chella [27] argues that the methodology used 
by Steels and Belpaeme is "a seminal starting point for the investigation of a general methodology for inferential learning of conceptual spaces from an agent's external perceptions, its inner and external constraints, and its actions." In Reference [28], I present another methodology, based on identifying invariances, for constructing conceptual spaces.

\subsection{Object Categories}

Next topic concerns how concepts in the form of object categories are represented. One property of an object is its location that is represented by ordinary space; other properties, such as color, size, weight, sound, and temperature, can be represented in different "category" domains, each with their own quality dimensions and geometric structures $[5,9]$. If the space domain represents where an object is, then the category domains represent what it is. ${ }^{3}$

As a paradigm example of an object category, consider $d o g$. When we learn the concept $d o g$ as children, among the domains that we first learn are presumably shape, size, sound, smell, color, and texture. Later, we learn about further domains of dogs such as their typical behavior (the action domain-see Section 2.5), biological characteristics, and their roles in human society. We also learn about the parts of a dog and how they are related (the meronomic structure).

Concepts for objects are not just bundles of properties. Since several domains are involved in a representation, some principle for how the different domains are to be weighed together must be assumed. The relative weights of the domains depend on the context in which the concept is used. For example, when packing eggs, their shape, size, and fragility are central domains; while in the context of cooking with eggs, their inner material, coagulation properties, and nutritional value come into focus. Hence, I assume that in addition to the regions associated with each domain, the object category representation contains information about the prominence of the different domains.

Object categories also contain information about correlations between regions from different domains that are associated with the concept. For example, the $d o g$ concept has a negative correlation between the size of the dog and the pitch of its barking, and for many fruits there is a correlation between its color and its sugar content. These considerations motivate the following definition for object categories [9]:

Criterion C: An object category is represented as a set of convex regions in a number of domains together with information about the prominence of the domains and about how the regions in different domains are correlated.

The set of domains that are used to represent object categories is open-ended and it can be expanded as the robot learns more about a category. For perceptual domains, the values for the dimensions can be obtained by various forms of robotic sensors or measuring methods. For example, Steels and Belpaeme [26] show how the color space can be generated from three input values for lightness, the red-green opponent channel, and the yellow-blue opponent channel. To this extent the meanings of the object categories are grounded in the external world [8, 29-31]. The object representation may, however, also contain non-perceptual dimensions; for example, kinship or ownership relations, the values of which must be obtained by indirect methods.

Furthermore, the borders of object categories may change over time as the robot learns about new types of examples as seen in Section 2.3. This entails that object categories cannot be defined in terms of necessary and sufficient conditions, which has been the standard method in traditional AI, but a characterization in terms of prototypes is more appropriate. Hence, a computational approach to a model of concepts and concept learning should take this into account.

\footnotetext{
${ }^{3}$ This distinction mirrors the processing of the dorsal and ventral streams of visual processing in the brain.
} 
Another aspect that is applicable for many object categories is the parts of the objects and their relations. A computational model that takes the meronomy of the object into consideration is presented in Reference [32]. They focus on two-dimensional shapes, but in principle their model can be extended to three dimensions. Fiorini et al. [33] show how such meronomic relations can be modeled in terms of conceptual spaces.

One should also consider how objects (or individuals, in the philosophical sense) can be represented in this framework. A straightforward way to represent an object is as a point in a conceptual space. Alternatively, a point in a conceptual space can be seen as a vector of coordinates, one for each dimension in the space. In this way, each object can be assigned a specific spatial position, color, shape, weight, temperature, and so on. Abstract entities have no location in space or time, so their underlying domains are different from those of physical objects.

Computationally, representing object categories becomes more complicated than representing properties. As regards the domains involved in an object category, the product space of them can be generated. Correlations between regions of the various domains are, however, more difficult to model in a programming-friendly way.

There exists a plethora of machine learning systems that generate information for object categorization-not only methods of deep learning, but also methods involving various sensors, artificial vision, and visual question answering systems (see References [34, 35] for surveys). Also, the area of visual scene description generating image captions (for example, Reference [36]) is advancing rapidly. The various methods can provide useful input to representations of objects and their properties in conceptual spaces.

Recently, systems developed for deep learning (for example, Reference [37]) have become popular. The systems can be trained to categorize different kinds of objects, sometimes more successfully than humans do it. The most pressing problem for this approach is that, unlike the Voronoibased method presented above, such systems require a very large training set to learn the relevant structures. Systems based on deep learning are useful for classificatory purposes, but it remains to be seen whether they can be developed to more general semantic structures of the type presented in this article. Because deep learning methods generally require a large number of training examples to produce reliable categorization, it is of interest to note that, once the dimensional structures are available to a robot, the method based on Voronoi tessellations that was outlined in Section 2.3 can be used to learn new categories using only a few training examples (see Reference [38] for some ideas about how to create transparent neural networks based on conceptual spaces).

\subsection{Actions}

So far, the examples of properties and object categories have all been of a static nature. However, it is obvious that a considerable part of representations necessary for the cognitive and communicative tasks of a robot concern dynamic concepts. Most importantly, a robot must be able to communicate about the actions of itself and of others.

When one perceives an action, one does not just see the movement, one also extracts the forces that control different kinds of motion. Runesson [39] claims that the kinematics of a movement contain sufficient information to identify the underlying dynamic force patterns. Following this idea, my proposal is that, by adding the force domain, one obtains the basic tools for analyzing the dynamic properties of actions [17, 40,41].

For many actions-for example, pushing and lifting-a single force vector may be sufficient, but for others-such as walking and swimming-a complex of forces is involved. I therefore define an action as a pattern of forces, since several force vectors are interacting (by analogy with Marr and Vaina's [42] system of differential equations). 
To identify the structure of the action space, one should investigate similarities between actions. For example, walking is more similar to running than to throwing. This can be accomplished by basically the same psychological methods used for investigating similarities between objects. In robotics, a common method for reducing the complexity of an action is to use a Kinect sensor that extracts the movements of a stick-figure representation of a person and then use a neural network to classify the reduced actions (e.g., References [43, 44]). An analogy between how objects and how actions are represented in conceptual spaces is that action concepts share a similar structure with object categories; in particular, they have prototypes [45]. Hence, I assume that the notion of betweenness is meaningful also in the action domain, allowing me to formulate the thesis that an action concept is represented as a convex region in the action domain $[2,40]$. Here, one may interpret convexity as the assumption that, given two actions in the region of an action concept, any interpolation of those actions will fall under the same concept. The geometric structure of the action domain is not well studied empirically, but in References [15] and [46] some results are presented.

In the psychological literature, there are some systems for categorizing human actions described by verbs, for example References [46] and [47]. These systems are psychologically motived, and they have not been developed with the aim of supporting human-robot communication. Recent computational work by Gharaee and her colleagues has resulted in an online system based on neural networks that can categorize and segment a number of bodily actions [43, 44, 48]. Such a system can be used by a robot to select a relevant verb (see Section 4.3) to be used in the robot's construction of sentences describing what is happening. For future developments of human-robot interaction it is important that efficient robot systems for action categorization become available.

Conceptual spaces were proposed (as an alternative to symbolic models and artificial neural networks) as a general framework for representing information [9]. It has been applied to a number of areas within AI and robotics (e.g., References [11, 38, 49-51]). In particular, Lieto et al. [38] propose that conceptual spaces "offer a lingua franca that allows to unify and generalize many aspects of the symbolic, sub-symbolic and diagrammatic approaches.” Despite such a general claim, one purpose of this article is to propose a more integrative semantic framework combining conceptual spaces with a model of event structure. This will be the topic of the following sections.

\section{THE STRUCTURE OF EVENTS}

Recent research indicates that much of human cognition is based on representations of events $[2,4,5,41,52]$. We use events in causal thinking, planning, and communication. Furthermore, our episodic memory depends on event structures [4]. The central idea of this article is that to communicate with humans in a natural manner, the semantics employed by a robot should also be based on event representations (see also Reference [53]). If the conceptual structures of the human and the robot do not match, then communication will be less efficient. In this section, I outline how conceptual spaces can be used to model events.

Three important notions can be defined very naturally in conceptual spaces:

- A state is a set of points in a conceptual space.

Borrowing the notion of state spaces in physics, each object and its properties is identified with a multi-dimensional point in a space.

A change of state is represented by a (nonzero) vector.

- The beginning of the (high-dimensional) vector represents the initial state and the end represents the result of the change.

- A path is a series of changes of states. 


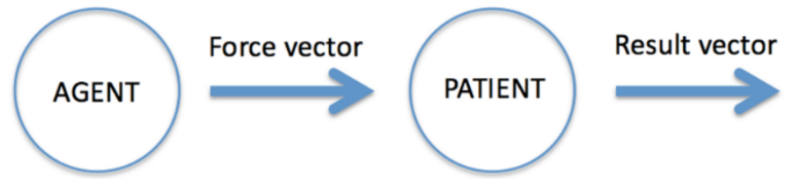

Fig. 4. The main components of an event representation.

In its original meaning, a path is a series of changes in physical space; but its meaning can naturally be extended to changes in more abstract domains.

A prototypical event $[2,41]$ is one in which the action of an agent generates a force vector (more generally, a force pattern) that affects a patient causing a change in the state of the patient (see Figure 4). More generally, an event is built up from an agent, an action, a patient, a result, and possibly other "thematic roles" such as instrument, recipient, and beneficiary [6,54]. Agent and patient are object categories with different properties. We assume that the agent is able to act, which in the proposed framework amounts to exerting forces. The core idea is that the representation of an event contains two vectors-the force of an action that drives the event and the result of the application of the force.

Following the previous section, an action is modeled as a force vector (or a pattern of force vectors as in walking). The result of an event is modeled as a change vector representing the change of properties of the patient before and after the event [5]. For example, when Oscar (the agent) pushes (the force vector) a table (the patient), the forces exerted make the table move (the result vector). Or when Victoria bends a stick, the result may be that the stick breaks. When the result vector is just a point-that is, when the result is no change-then the event is a state.

More formally, events can be described as a product of two vector spaces. The first is a force vector (or several force vectors), representing the action part of an event, and the second is a result vector. The second vector describes either a movement in space, in which case the space domain is involved, or a change of properties in the object involved (the patient of the event). In this case, the vector is modeled with the aid of a domain among the category domains or some other domain. The upshot is that events connect the domains of space, object categories, and actions.

A special case of the event model, expressed linguistically by intransitive constructions such as "Victoria is walking" and "Oscar is jumping," is when the patient is identical with the agent. In this case, the agent exerts a force on itself. In other words, the agent modifies its own position in some domain of agent space (= patient space).

The event model captures a basic sense of causation: The action of the agent causes the change in the patient. The model captures causation by introducing a distinction between forces and changes of states $[55,56]$. Where there is an effect, there is a cause. In other words, if the result vector of an event is nonzero, the force vector must also be nonzero.

The mapping from the force vector to the result vector is the part that is most difficult to implement computationally. Such a mapping represents the robot's expectations concerning the consequences of various actions. In general, however, such expectations are dependent on the context. If the result is a movement in space, then some form of physical simulator may be used, but for result vectors in other domains, specialized models may be required.

\section{THE SEMANTIC STRUCTURE OF WORD CLASSES}

In this section, I show that the structures building on conceptual spaces that have been presented in Sections 2 and 3 can be used in a constructive and natural way to generate semantic models of the major word classes. The models are sufficiently precise to be implemented in a robot (an implementation, based on Reference [57], will be presented in Section 5.4). 


\subsection{Nouns}

In Section 2.4, I introduced object categories as concepts that involve several domains (in contrast to properties). Object categories are expressed linguistically by nouns such as dog, apple, and chair. I take such categories as the most typical meaning of nouns. The first nouns that children learn are of this type.

To be sure, there are nouns that refer to many other kinds of entities. For example, abstract nouns have no physical referents, for example law, retirement, inflation, and mind. Lyons [58], pp. 442445] distinguishes three fundamental "orders": (1) physical objects; (2) events, processes, states-ofaffairs; and (3) propositions, schemas, and so on that "are outside space and time." ${ }^{*}$ The nouns in (2) and (3) require other types of meaning domains than those used for the object categories in (1). Here, I will not analyze these nouns, since they are less central for human-robot communication.

But even among nouns expressing physical objects, one can find different types. An important semantic distinction is that between mass nouns such as silver, sand, and water and count nouns that refer to countable objects such as car, house, and flower. Some nouns can be used in both ways: Compare "There are five apples in the bowl" (count use) with "Put two cups of apple on top of the pie dough" (mass use). My account for the mass-count distinction is that for mass nouns the material domain becomes the most prominent one-the shape domain is of minor importance. For example, apple as a mass noun just concerns the material. For count nouns, it is the other way around. This is an example of how varying the prominence of domains may change the meaning of a word.

\subsection{Adjectives}

My account of the semantics of adjectives will be based on my notion of properties as formulated in Criterion P. The key idea is that adjectives express properties. This motivates the following thesis $[5,9]$ :

Single-domain thesis for adjectives: The meaning of an adjective can be represented as a convex region in a single domain.

For many adjectives, the single-domain thesis is valid. In particular, I have argued [9] that all color terms in natural languages express convex regions with respect to the color domain. This means, for example, that there should be no language that has a single word for the colors denoted by "green or orange" (and which includes no other colors), since such a word would represent two disjoint areas in the color domain. Strong support for this conjecture has been obtained by Jäger [13] (see also References [59, 60]). Furthermore, there is, for example, no adjective that means "long and hot," since such a word would involve two different domains.

Adjectives are also used for comparing things: Most languages have comparatives such as taller and smarter. Many languages also have superlatives; for example, tallest and smartest, which can be defined from comparatives. The meanings of these adjectives are easy to implement computationally using a one-dimensional domain.

\subsection{Verbs}

In Section 3, I proposed that when we describe an event at least one of the force and result vectors and at least one of the patient and the agent are parts of the description [5, 41]. When describing an event, agents and patients are typically expressed by noun phrases and actions and results by verb phrases. Given this suggestion for event expressions, the model thus explains the basic distinction

\footnotetext{
${ }^{4}$ See References [61] and [62] for finer classifications of nouns.
} 
between nouns phrases and verb phrases. In contrast to mainstream linguistics, this distinction is made on a semantic basis derived from the cognitive representation of events.

My analysis of verbs begins from event representations, and the aim is to identify meaning constraints for verbs. I will focus on the meanings of verb roots, since the variety of possible syntactic modifications would make a full semantic analysis of verbs very complicated. The key idea for the semantics of verbs is the following [5]:

Single-domain thesis for verbs: A verb root refers either to the force vector or to the result vector of an event.

For example, push refers to the force vector of an event (and thus the force domain), move refers to changes in the spatial domain of the result vector, and heat refers to changes in the temperature domain. ${ }^{5}$ The single-domain thesis for verbs is analogous to the single domain thesis for adjectives. The thesis entails that there are no verbs that mean "walk and burn" (multiple domains) and there are no verbs that mean "crawl or run" (not convex).

Linguists distinguish between manner verbs and result verbs-where "manner verbs specify as part of their meaning a manner of carrying out an action, while result verbs specify the coming about of a result state" ([63], p. 21; see also Reference [64], Ch. 1).

The single domain thesis for verbs entails that manner verbs refer to force vectors of events while result verbs refer to result vectors. Another way of expressing this is to say that the manner/result distinction is basically a cause/effect distinction: Manner verbs refer to causes and result verbs to effects.

In the context of robotics, research has focused on how result verbs can be modeled (e.g., References [65-68]. However, when it comes to human-robot interaction, the robot should also be able to recognize human actions by the manner they are performed. This is often called recognition of biological motion [45]. Recognizing actions is important in particular if the robot is supposed to model the intentions of a human. For example, it can be important to distinguish a human waving "come" from just a social waving or from chasing away mosquitos.

A direct consequence of the single-domain thesis is that the distinction between the different kinds of verbs is determined by the domain associated with a verb. If the domain is that of the force patterns underlying actions, then it is a manner verb. If it is the spatial domain or an object category domain, then it is a result verb. Thus, the single-domain thesis together with the classification of domains can explain why these two kinds of verbs fall out as natural classes.

\subsection{Prepositions}

Most prepositions can be grouped into two classes: locative, indicating where something is, and directional, indicating where something is going. Locative prepositions specify the location (a region) in the spatial domain: "Give me the bottle behind the bread!" Another function is fulfilled by directional prepositions. In a sentence such as "Oscar went to the library," the phrase "to the library" has the same semantic function as a result verb: it specifies the result vector of an event. My proposal is that locative prepositions are represented by convex sets of points and directional prepositions by convex sets of paths. In line with the analyses of the semantics of adjectives and verbs, I therefore put forward the following thesis:

Single-domain thesis for prepositions: Prepositions represent convex sets of points, paths, or vectors in a single domain.

\footnotetext{
${ }^{5}$ Possible exceptions to this general rule are verbs that describe changes in ontology and verbs like give that describe intentional actions involving recipients. These are discussed in Reference [5, Section 10.3.2].
} 
Locational and directional prepositions depend on the spatial domain. In general, this domain is represented with the aid of the Cartesian coordinates representing width, depth, and height and where distances are measured using a Euclidean metric. However, there is another way of representing space; namely, in terms of polar coordinates that represent points in space in terms of distance from an origo and horizontal and vertical angles. A new definition of "between," and thereby convexity, that differs from standard Euclidean betweenness can be defined in terms of polar coordinates (see Reference [5], Ch. 11, and Reference [69] concerning the technical details). For example, the set of points of a semicircle form a convex set with respect to polar convexity. Given this definition of convexity, it can be shown that most locative prepositions, such as inside, outside, near, far, in front of, and behind, can be represented by a convex set of points.

Similarly, a betweenness relation for paths is easy to define [69] and thereby convexity of sets of paths. On the basis of such a definition, it is easy to show that the meaning of directional prepositions-for example, to, from, into, out of, through, along, and across-correspond to convex sets of paths. For example to and into can be defined by using locational prepositions, as the following sets of paths where $\mathrm{p}(0)$ is the beginning of a path and $\mathrm{p}(1)$ its end:

to $=\{p:$ near $(p(1))$ and not near $(p(0))\}$

into $=\{p$ : inside $(p(1))$ and not inside $(p(0))\}$

In contrast to most analyses within linguistics, I argue that prepositions do not only refer to the spatial domain. Some prepositions refer to the time domain. In English, the most common ones are before and after. In addition, most typical uses of the prepositions against, over, on, and in depend on the force domain (see Reference [5], Ch. 11, and Reference [70] for arguments).

\subsection{Adverbs}

In the semantic model of verbs presented in Section 4.3, verbs refer to vectors. Vectors can vary in terms of dimension, orientation, and magnitude. Therefore, adverbs that are modifiers of verbs should refer to change in these features. For example, in "I speak slowly," the adverb selects one of the several dimensions from the sound domain of speak. "I speak loudly" selects another. In "I walked backwards" the adverb refers to the orientation of my motion. Finally, in "Victoria pushed the door softly," the magnitude of the force vector representing push is diminished by the adverb. When an action involves a pattern of forces, adverbs can modify the whole pattern by providing dynamic information; for example, "Victoria walked limply," "Oscar smiled wryly," or "Victoria kicked aggressively." What is common to these examples is that the adverb restricts the regions associated with the meanings of the manner verbs. Similarly, in relation to a result verb describing a concatenation of changes (as in a path), an adverb can provide information about the form the path takes; for example, "Victoria crossed the football pitch crookedly." In brief, the function of adverbs modifying verbs is parallel to how adjectives modify nouns.

As long as adverbs function as multipliers (diminishers or magnifiers) within a particular domain, the convexity principle can be defended. For example, if certain voice volumes $\mathrm{v}_{1}$ and $\mathrm{v}_{2}$ both count as speaking loudly, then any volume between $\mathrm{v}_{1}$ and $\mathrm{v}_{2}$ will also count as loudly. And for adverbs expressing the form of a path, the principles of path convexity that were mentioned in Section 4.4 will apply. It is an open question whether the convexity principle can also be applied to other adverbs.

\subsection{Deictic Expressions}

Gärdenfors and Brala [71] extend the single domain hypothesis to demonstratives and articles. They show that the meanings of demonstratives and articles can be analyzed in terms of a combination of the spatial domain and a fixed set of semantic domains. For example, the demonstratives here and there refer to regions of physical space, while this and that refer to a combination of 
physical space and the object category space. The basic communicative function of demonstratives and articles is to help achieve joint reference among interlocutors by directing referential processes toward the discourse referent in the external environment or in the mutually shared mental space (the "common ground" in the terminology of Clark [72]). Although deictic expressions are obviously important in HRC, they have received very little attention in research.

To sum up, in this section I have shown that convex sets and vectors are unifying tools for modeling the semantics of the most important word classes. The single domain hypotheses that apply to all the word classes discussed, except for nouns, generate strong constraints on the meanings of different types of words. These hypotheses are defended in Reference [5]. To the extent that the conceptual domains used to represent the meaning are connected to actions or perception, the semantics will be grounded in the sense of Reference [8] (see also References [1, 29, 31, 73-76]).

\section{FROM EVENT STRUCTURE AND WORD MEANINGS TO SENTENCES}

Most research on the use of language in human-robot interaction has focused on how a robot can understand commands given in natural language by a human. For many practical applications of robots, the communicative demands involve a more dialogic form, containing also declarative sentences and questions produced both by the human and the robot. The framework presented above has the potential to also cover such forms of communication. In this section, I will focus on the problem of how a robot can generate relevant sentences, since this aspect has been less studied in HRC. However, the semantic framework can also be used in the process of understanding sentences.

\subsection{Generating Sentences}

Communication does not only depend on the meaning of single words, but importantly on how words can be combined to express desired messages. Hence, for successful robot-human communication, the robot must be able to compose words, in particular in the form of sentences.

The problem to be discussed in this section is how the robot's representation of an event can be turned into a sentence describing aspects of the event that are relevant to the communicative situation. Not only what they perceive, but also what the robot and the human are attending to, their current goals, their future plans, and their previous communication will influence the sentence production. The focus in HRC has been on how a robot can understand a sentence; in particular, a command produced by a human (see, e.g., References [31, 77-79]). Here I instead consider the problem of how a robot can generate sentences that fulfill the goals of the robot. Even though the two problems are related, the event semantics becomes more central when generating sentences. Another difference is that in the works of Matuszek, Tellex, and their colleagues [31, 77-79], words are given meaning by being grounded in the perceptions and actions of the robot. In the framework proposed here, words are given meaning by being mapped onto regions of conceptual spaces. This means that, unlike the command situations, sentences can be used to express information about objects or individuals that are not present in the context. Such "displaced" uses of language are one of its main characteristics [80]. As a result, the human-robot communication is not always dependent on the surrounding scene. However, the meanings of most words must be grounded via their corresponding perceptual domains. In brief, an artificial system that does not have a sufficiently rich equipment of sensors will communicate in a parrot-like fashion. ${ }^{6}$

In their review of natural language generation in computational systems, Gatt and Krahmer (Reference [81], p. 70) separate six steps:

\footnotetext{
${ }^{6}$ This is the case for most so-called chatbots.
} 
- Deciding which information to include in the language output under construction

- Determining in which order information will be presented in the output

- Deciding which information to present in individual sentences

- Finding the right words and phrases to express information

- Selecting the words and phrases to identify domain objects

- Combining all words and phrases into well-formed sentences.

Here I focus on the first step and make some comments on the second and the third, but I do not consider the remaining steps.

\subsection{Construals}

A naïve understanding of language production is that when we see an event, we extract its meaning. Events are, however, complex phenomena and they cannot be exhaustively described. The first step in generating a sentence is thus to decide which information to include. One of the great liberties in language use is the ability to express different perspectives that correspond to different meanings derived from the same event. One manner by which different perspectives can be expressed is via different grammatical constructions, where constructions represent the correspondence between sentence form and meaning. For example, in "Victoria paints Oscar's face," Victoria is more in focus; while in the passive version, "Oscar's face is painted by Victoria," the focus is on Oscar's face. From the point of view of implementation, it is important to distinguish two different levels of meaning: (1) the representation of an event and (2) a construal that picks out certain aspects of the event. The construal is what is then expressed in a sentence.

There are, however, several aspects of how a construal is formed (see Reference [82], Ch. 3, and Reference [83], Ch. 3, for a survey). The attention of the speaker is central in forming a construal. By analogy with the visual process-where we can only focus our attention on some features of the visual field-a construal focuses only on certain parts of an event. The sentences "Oscar sprayed paint on the wall" and "Oscar sprayed the wall with paint" describe the same event with the aid of two different construals. The difference between them is that in the first, "paint" is focused on as the patient of the action, while in the second "the wall" is made the patient ([54], p. 124).

Another aspect of a construal is perspective: For example, if I and you are located on two sides of a house, I can say that you are behind the house, if I put myself in the center, or I can say that you are in front of the house, if I put the house and the direction of its main side in focus. Yet another aspect is categorization: A construal must select a level of generality to describe an object; for example, terrier, dog, mammal, or animal.

In Reference [5], Chapter 11, I have proposed the following thesis:

Thesis about event construals: A construal of an event contains at least one vector (force or result) and at least one object (patient or agent).

I define a construal as such a mental model of an event together with a particular focus of attention added to it (see, e.g., Reference [3], Section 1.4, Reference [84], Section 3.3, and Reference [85]. The application of an attention process selects the force or the result vector, generating a construal that can be used in the language production model.

\subsection{From Construals to Sentences}

On the basis of the thesis about event construals, one can describe a fundamental connection between the semantics of sentences and events: 
Thesis about sentences: A (declarative) sentence expresses a construal of an event. ${ }^{7}$

This thesis implies that at least an agent or a patient (expressed by a noun phrase) and a force vector or a result vector (expressed by a verb phrase) are parts of what is expressed. Thus, the two main components noun phrase and verb phrase have to be present in a linguistic description of an event. Consequently, the model of events and the proposal about construals explain the central components of a sentence. The structures thereby provide a motivation for why a sentence is a cognitive unit of communication.

Given a particular perspective of an event that the robot wants to express, it should generate a sentence that produces that meaning in the listener. A given event can be described from different perspectives, and so the problem of characterizing the corresponding meaning from a scene is not trivial $[41,86]$.

Consequently, no simple mapping exists between the role taken in an event and the designation of subject, object, or oblique in a sentence. In English (and many other languages), the most focused role is designated subject and the secondary focus is designated object. In agreement with Givón (Reference [85], p. 198), I see the selection of focus not as directly part of event representation, but rather as a central element of the construal process. This setup avoids the problems that arise when event representation and construals are conflated. Robots have conversational goals in producing construals. Consequently, the construals are contextual, depending on what the human conversation partner already knows or believes or will find most interesting and what the robot wants to draw attention to.

For example, consider an event where Victoria (agent) scrubs (action) a table (patient) clean (result). This event can result in a number of different sentences that all fall under the thesis about construals of events (it is also marked which type of verb is used ${ }^{8}$ ):

"Victoria scrubs" (agent, action, intransitive)

"Victoria cleans" (agent, result, intransitive)

"The table was cleaned" (patient, result, passive)

"The table was scrubbed" (patient, action, passive)

"Victoria scrubs the table" (agent, action, patient, transitive)

"Victoria cleans the table" (agent, result, patient, transitive)

"Victoria scrubs the table clean" (agent, action, patient, result, transitive)

Different languages have different preferences for which construals are expressed. For so-called verb-framed languages [87], for example Romance languages, agent-result-patient constructions are the most typical. An example from Spanish is "La botella entra en la cueva" (the bottle enters the cave). However, so called satellite-framed languages, for example English and Germanic languages, agent-action-patient constructions are preferred. The event above is typically described in English as "The bottle is floating into the cave." In the last example, the preposition into expresses the result vector. If one wants to add the action to the Spanish example, then it becomes "La botella entra en la cueva flotando" (the bottle enters the cave floating).

Given the thesis about event construals, a robot needs at least the following to generate a sentence: (1) identifying an agent or a patient or both; and (2) identifying an action or a result vector or both. Agents and patients belong to object categories. As mentioned earlier, there are several techniques available for identifying such categories, so I will not discuss that step. A less standard problem is how to identify the action that makes something an agent. However, in human-robot

\footnotetext{
${ }^{7}$ Exceptions to this thesis are generic sentences such as "Kangaroos are marsupials." They have a different function in communication in that they propose an update of the underlying semantic structure.

${ }^{8}$ Scrub and clean can be used both intransitively and transitively.
} 
interaction, the relevant agent is typically a human, which means that the problem in many cases can be reduced to categorizing the action of the human. This amounts to identifying the force patterns exercised by the human. If the robot is asking the human to do something, then it can construct a request using a manner verb that captures the force pattern that will lead to the desired result.

Similarly, the robot must identify the patient; that is, the object towards which the force pattern is directed. Finally, the robot must identify the relevant change of some property of the patient; for example, that it moves, that it is heated, that it is painted, or that it is destroyed. This entails that the robot must take a dynamic perspective and should compare the properties of the patient before and after the action is performed.

I have no universal solution to the programming problems involved in the process of generating a construal that I have outlined here. I believe, however, that the problems are tractable for many types of human-robot interaction, and the next subsection will present an implementation. The event perspective on sentence generation will help identify which actions are important and which changes of properties should be focused on. The basic event constituents-agent, patient, action, and result-can be complemented with other thematic roles such as recipient ("Oscar handed Victoria a towel") and instrument ("Oscar painted the house with a roller"). Here I will, however, not discuss how such extensions can be implemented computationally.

\subsection{An Implementation for an iCub Robot}

The system implemented for an iCub robot, described in Reference [57], is based on the "reservoir computing" in neural networks developed by Dominey and his colleagues (for example, References $[67,88])$. The elements in the reservoir are sorted into information about three thematic roles: agent, patient (object), and recipient, together with a "predicate" that corresponds to the information given in a verb (sometimes a prepositional phrase). The agents are the iCub and humans, and the patients are objects that are located on the table between the human and the iCub. The objects can be identified by the iCub. The predicates are divided into manner verbs such as "push," "grasp," "point," and result verbs such as "move," "hold," and "has."

The central components of the system are a sentence comprehension model that assigns thematic roles to the elements of a sentence, and a sentences production model where a coded meaning is turned into a sentence with the aid of the neural network. Here I focus on the production model. In addition, there is an "object properties collector" that keeps track of the objects and their properties. The system also contains a spoken language platform that allows a naturalistic dialogue between the human and the iCub. For details, see Reference [57].

The production model follows the division between focusing on the action or on the result of an event. In the implementation, the choice is driven by the type of question given by the human that controls the attention of the robot. For example, if the human asks the iCub "What did you do?", the robot will create a construal of a sentence that focuses on the force vector of the event so a manner verb is selected; for example, "I pushed the cube." However, if the human asks "What happened?", the focus will be on the result and the iCub may answer "The cube moved to the left." The functional architecture of the system is presented in Figure 5.

In the implementation, only the actions of the iCub are considered, and they form the basis for the manner verbs that are used in the dialog. However, since the iCub can be combined with a Kinect camera, it is possible to extend the system to also allow actions performed by humans to be coded by using some action categorization system that works in real time (for example, Reference [43]). This would allow a richer communication between the iCub and humans.

\footnotetext{
${ }^{9}$ For a video of the interaction, see http://youtu.be/7aeArAn7yLo.
} 


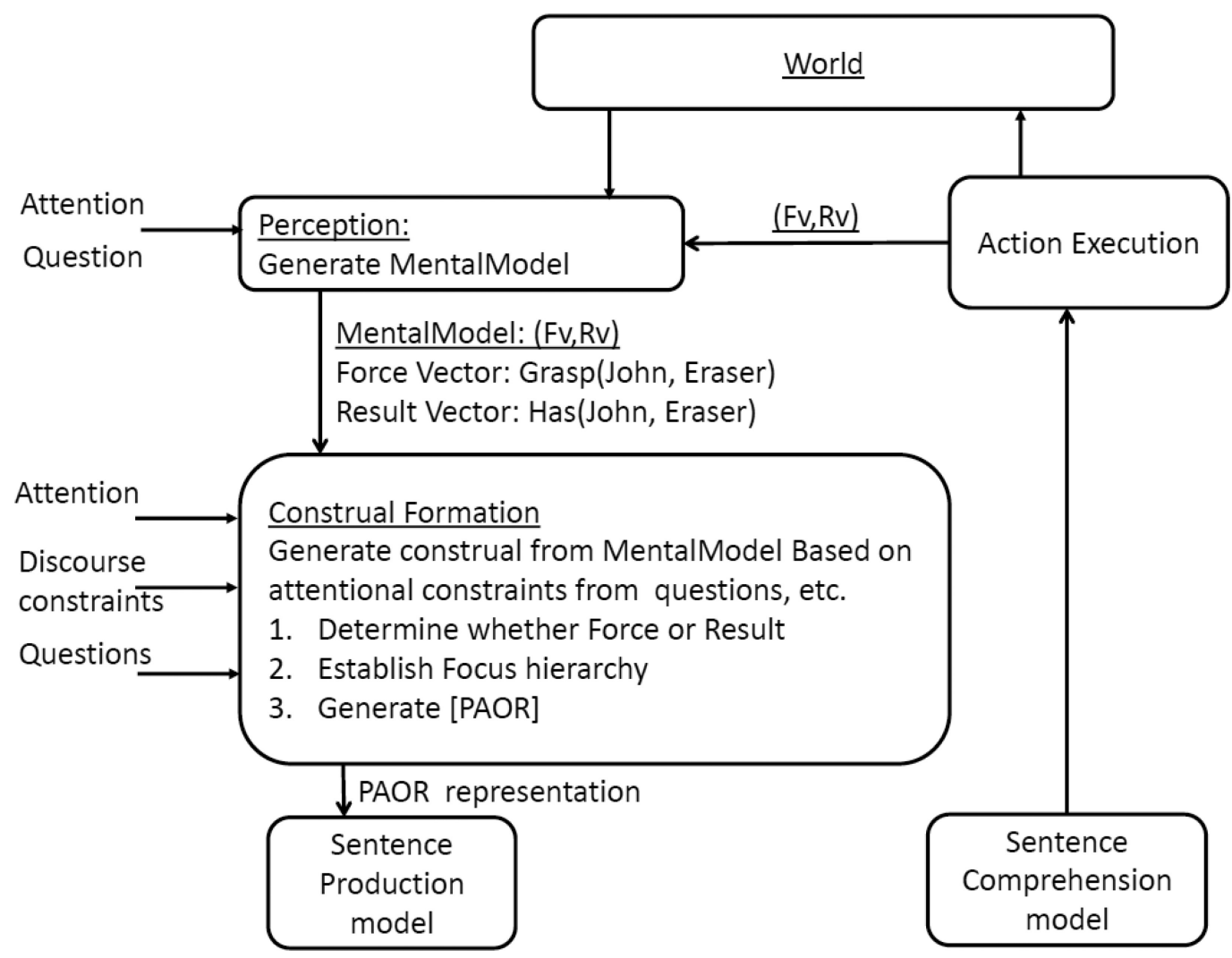

Fig. 5. The functional architecture of the iCub dialog system (from Reference [57], p. 61). Fv = force vector, $\mathrm{Rv}=$ result vector, $\mathrm{PAOR}=$ Predicate, Agent, Object or patient, Recipient. Reprinted with permission from John Benjamins Publishing Company.

\subsection{Other Approaches}

To be sure, there are other approaches to how a robot can learn semantic representations for human-robot communication. Here I will only consider two language learning models that are related to my proposed forms of representations (other methods have been studied by Matuszek and colleagues [77, 78]).

The first approach has been developed within the iTALK (Integration and Transfer of Action and Language Knowledge in Robots) project [63, 75, 89]. The model is inspired by infant development up to 2 years of age. It focuses on (1) infants learning about controlling their bodies and the environment, (2) their social learning, and (3) the development of their linguistic capability. Via associative learning using neural networks built from self-organizing maps, the iCub learns words for the shape (expressed by a noun) and color (expressed by an adjective) of a number of objects. The iCub is then programmed to perform a small set of actions on the objects expressed by verbs such as take, push, touch, point at, look at, move left/right. However, the robot cannot interpret the actions of a human. The verbal interaction consists of spoken commands from the human; for example, "push the blue octopus." The iCub understands certain forms of spoken language, but only produces rudimentary speech itself-it just repeats the color and the shape words for the object involved in the action. This means that there is no analysis of the thematic roles of the entities 
involved in the interaction, let alone of the event structure. Although the robot solves several perceptual categorization problems related to the interaction, including interpreting spoken language and the color and shape of objects, the semantic structures it uses are still relatively simple.

The second approach developed by Tellex and colleagues [31,79] consists of a model for understanding natural language commands given to a robotic system (a fork-lift) that navigates and manipulates objects. Similar to the system of Mealier et al. [57], it also exploits thematic roles in the analysis of the command sentences. The thematic roles are used to (probabilistically) derive spatial description clauses using syntactically oriented "generalized grounding graphs." This is in contrast to the semantic framework presented in this article that is almost independent of syntax. The graph that is evaluated as having the most probable grounding in the scene is then used to generate the action sequence for the robot.

The system is limited to understanding commands, and there is no sentence generation or any other form of dialogic elements. What is called events in Reference [79] correspond to what is here analyzed as result vectors. Consequently, there are no manner verbs describing actions that are included in the commands. Furthermore, there is no mechanism that controls the attention of the robot.

\section{CONCLUSION: A ROBOT THAT UNDERSTANDS THE MEANING OF WORDS AND KNOWS WHAT TO SAY}

The aim of this article has been to present semantic representations that are suitable for robothuman communication. The new idea is to use the structure of events for this purpose. This idea is supported by current research concerning human cognition. The event semantics outlined in this article can be used for many purposes, not just generating or understanding sentences. It can also be applied in causal reasoning and problem solving and thereby become a central component in the robot's planning module. For example, Voronoi tessellations have also been used in pathplanning algorithms [90]. The event semantics thereby forms a general representational structure for how knowledge is handled in a robot. Current methods based on neural networks do not allow such a general framework.

I have focused on the two-vector model of events [2,5] and how it can be interpreted with the aid of conceptual spaces [9]. This model naturally generates the most central thematic roles studied by linguists. Then I have shown how the vectors and the thematic roles can be used to specify the specific semantics features of various word classes in a way that is implementable in a robot system.

I have also presented a method based on event structures for putting words together into sentences. The focus has been on selecting the components to be used in the output-the construaland the computational problems involved in this process. I have put less emphasis on the grammatical structure of the output, since this is, in my opinion, a secondary problem in human-robot communication.

Gatt and Krahmer (Reference [81], p. 80) write that there is no systematicity in the various approaches to natural language generation in AI. They note that many application domains are small and the variation of expression is minimal. In these cases, outputs are generally specified using templates. They note that " $[t]$ he disadvantage of templates is that they are labour-intensive if constructed by hand." Even though templates can sometimes be learned automatically from corpus data, this method does not scale well to broader problem areas. The approach to language generation based on conceptual spaces and event structures that I have presented in this article offers a more general avenue. The approach has been implemented by Mealier et al. [57] for a limited set of verbs, but a more general implementation still remains to be developed. 


\section{REFERENCES}

[1] T. Taniguchi, T. Nagai, T. Nakamura, N. Iwahashi, T. Ogata, and H. Asoh. 2016. Symbol emergence in robotics: A survey. Adv. Rob. 20 (2016), 706-728.

[2] P. Gärdenfors and M. Warglien. 2012. Using conceptual spaces to model actions and events F. Sem. 29 (2012), 487-519.

[3] W. Croft. 2012. Verbs: Aspect and Argument Structure. Oxford: Oxford University Press.

[4] G. A. Radvansky and J. M. Zacks. 2014. Event Cognition. Oxford: Oxford University Press.

[5] P. Gärdenfors. 2014. The geometry of meaning: Semantics based on conceptual spaces. The MIT Press, Cambridge, MA.

[6] D. Dowty. 1991. Thematic proto-roles and argument selection. Language 67, 547-619.

[7] P. Gärdenfors. 2014. The evolution of sentential structure, Hum. Mente f. Philos. Stud. 27 (2014), 79-97.

[8] S. Harnad. 1990. The symbol grounding problem. Physica D. 42, 335-46.

[9] P. Gärdenfors. 2000. Conceptual Spaces: The Geometry of Thought. The MIT Press, Cambridge, MA.

[10] R. N. Shepard. 1982. Geometrical approximations to the structure of musical pitch. Psych. Rev. 89 (1982), $305-333$.

[11] P. Gärdenfors. 2004. How to make the semantic web more semantic. In Formal Ontology in Information Systems, A. C. Varzi and L. Vieu (Eds.). IOS Press, 19-36.

[12] P. Gärdenfors. 1990. Induction, conceptual spaces, and AI. Philos. Sci. 57, (1990), 78-95.

[13] G. Jäger. 2010. Natural color categories are convex sets. Amsterdam Colloq. 2009, LNAI 6042, 11-20.

[14] G. Fairbanks and P. Grubb. 1961. A psychophysical investigation of vowel formants. F. Speech Hear. Res. 4 (1961), 203-219.

[15] B. Malt, E. Ameel, M. Imai, S. Gennari, N. M. Saji, and A. Majid. 2014. Human locomotion in languages: Constraints on moving and meaning. Mem. Lang. 74 (2014), 107-123.

[16] P. Gärdenfors. 2001. Concept learning: A geometrical model. Proc. Aristotelian Soc. 101 (2001), 163-183.

[17] M. Warglien and P. Gärdenfors. 2013. Semantics, conceptual spaces, and the meeting of minds. Synthese, 190 (2013), 2165-2193. DOI : $10.1007 / \mathrm{s} 11229-011-9963-\mathrm{z}$

[18] E. Rosch. 1975. Cognitive representations of semantic categories. F. Exper. Psychol.: Gen. 104 (1975), 192-233.

[19] C. Mervis and E. Rosch. 1981. Categorization of natural objects. Ann. Rev. Psychol. 32 (1981), 89-115.

[20] A. Okabe, B. Boots, and K. Sugihara. 1992. Spatial Tessellations: Concepts and Applications of Voronoi Diagrams. John Wiley \& Sons, New York.

[21] I. Douven, L. Decock, R. Dietz, and P. Égré. 2013. Vagueness: A conceptual spaces approach. f. Philos. Log. 42 (2013), 137-60.

[22] R. M. Nosofsky. 1988. Similarity, frequency, and category representations, f. Exper. Psychol.: Learn., Mem. Cog. 14 (1988), 54-65.

[23] A. Aamodt and E. Plaza. 1994. Case-based reasoning: Foundational issues, methodological variations, and system approaches. AI Commun. 7, 1 (1994), 39-59.

[24] P. Langley. 1996. Elements of Machine Learning. Morgan Kaufmann, San Francisco, CA.

[25] I. H. Witten, E. Frank, M. A. Hall, and C. J. Pal. 2016. Data Mining: Practical Machine Learning Tools and Techniques. Morgan Kaufmann, Burlington, MA.

[26] L. Steels and T. Belpaeme. 2005. Coordinating perceptually grounded categories through language: A case study for colour. Behav. Brain Sci. 28, 4 (2005), 469-488.

[27] A. Chella. 2005. How to learn a conceptual space. Behav. Brain Sci. 28, 4 (2005), 492.

[28] P. Gärdenfors. 2018. From sensations to concepts: A proposal for two learning processes. Rev. Philos. Psychol., 1-24. DOI : $10.1007 / \mathrm{s} 13164-017-0379-7$

[29] P. Gärdenfors and M.-A. Williams. 2003. Building rich and grounded robot world models from sensors and knowledge resources: A conceptual spaces approach. In Proceedings of the Autonomous Minirobots for Research and Edutainment Conference (AMiRE'03). 123-132.

[30] M.-A. Williams, J. McCarthy, P. Gärdenfors, C. Stanton, and A. Karol. 2009. A grounding framework. F. Autonom. Agents Multi-Agent Syst. 19 (2009), 272-296.

[31] S. Tellex, T. Kollar, S. Dickerson, M. R. Walter, A. G. Banerjee, S. Teller, and N. Roy. 2011. Understanding natural language commands for robotic navigation and mobile manipulation. In Proceedings of the 25th AAAI Conference on Artificial Intelligence. 1507-1514.

[32] S. C. Zhu and A. L. Yuille. 1996. FORMS: A flexible object recognition and modelling system. Int. f. Comput. Vis. 20 (1996), 187-212.

[33] S. Fiorini, P. Gärdenfors, and M. Abel. 2014. Structure, similarity and spaces. Cog. Proc. 15 (2014), 127-142.

[34] K. Bernard. 2016. Computational Methods for Integrating Vision and Language. Morgan and Claypool Publishers, San Rafael, CA.

[35] R. Bernardi, R. Cakici, D. Elliot, E. Erdem, N. Ikizler-Cinbis, F. Keller, A. Muscat, and B. Plank. 2016. Automatic description generation from images: A survey of models, datasets, and evaluation measures. 7. Artific. Intell. Res. 55 (2016), 409-442. 
[36] M. Mitchell, J. Dodge, A. Goyal, K. Yamaguchi, K. Stratos, X. Han, A. Mensch, A. Berg, X. Han, T. Berg, and H. Daume III. 2012. Midge: Generating image descriptions from computer vision detections. In Proceedings of the Conference of the European Chapter of the Association for Computational Linguistics (EACL'12). 747-756.

[37] J. Schmidhuber. 2015. Deep learning in neural networks: An overview. Neural Networks 61 (2015), 85-117.

[38] A. Lieto, A. Chella, and M. Frixione. 2018. Conceptual spaces for cognitive architectures: A lingua franca for different levels of representation BICA F. 19 (2017), 1-9.

[39] S. Runesson. 1994. Perception of biological motion: The KSD-principle and the implications of a distal versus proximal approach. In Perceiving Evens and Objects, G. Jansson, S.-S. Bergström, and W. Epstein (Eds.). Lawrence Erlbaum, Hillsdale, NJ, 383-405.

[40] P. Gärdenfors. 2007. Representing actions and functional properties in conceptual spaces. Body, Language and Mind, Volume 1: Embodiment, T. Ziemke, J. Zlatev, and R. M. Frank (Eds.). Mouton de Gruyter, Berlin, 167-195.

[41] M. Warglien, P. Gärdenfors, and M. Westera. 2012. Event structure, conceptual spaces and the semantics of verbs. Theor. Ling. 38 (2012), 159-193.

[42] D. Marr and L. Vaina. 1982. Representation and recognition of the movements of shapes. Proc. Royal Soc. London, B214 (1982), 501-524.

[43] Z. Gharaee, P. Gärdenfors, and M. Johnsson. 2016. Action recognition online with hierarchical self-organizing maps. In Proceedings of the 12th International Conference on Signal, Image Technology and Internet Based Systems. IEEE, 538544. DOI : 10.1109/SITIS.2016.91

[44] Z. Gharaee, P. Gärdenfors, and M. Johnsson. 2017. Hierarchical self-organizing maps system for action classification. In Proceedings of the International Conference on Agents and Artificial Intelligence (ICAART'17). 583-590. DOI : 10.5220/ 0006199305830590

[45] P. Hemeren. 2008. Mind in Action: Action Representation and the Perception of Biological Motion, Lund University Cognitive Studies, 140.

[46] M. Giese and M. Lappe. 2002. Measurement of generalization fields for the recognition of biological motion. Vis. Res. 42 (2002), 1847-1858.

[47] M. A. Giese, I. Thornton, and S. Edelman. 2008. Metrics of the perception of body movement. F. Vis. 8 (2008), 1-18.

[48] Z. Gharaee. 2018. Recognizing human actions by a multi-layer growing grid architecture. In Proceedings of the International Conference on Neural Networks (ICNN'18).

[49] A. Chella, M. Frixione, and S. Gaglio. 1997. A cognitive architecture for artificial vision. Artific. Intel. 89 (1997), $73-111$.

[50] F. Kaplan. 2000. L'émergence d'un lexique dans une population d'agents autonomes. These de Doctorat de l'Université Paris VI

[51] A. Chella, S. Gaglio, and R. Pirrone. 2001. Conceptual representations of actions for autonomous robots. Rob. Autonom. Syst. 899 (2001), 1-13.

[52] J. M. Zacks, N. K. Speer, K. M. Swallow, T. S. Braver, and J. R. Reynolds. 2007. Event perception: A mind-brain perspective. Psychol. Bull. 133, 2 (2007), 273-293.

[53] P. Gärdenfors, M.-A. Williams, B. Johnston, R. Billingsley, J. Vitale, P. Peppas, and J. Clark. 2018. Event boards as tools for holistic AI. To appear in the Proceedings of the International Workshop on Artificial Intelligence and Cognition (AIC'18).

[54] B. Levin and M. Rappaport Hovav. 2005. Argument Realization. Cambridge University Press, Cambridge, MA.

[55] P. Wolff. 2007. Representing causation. J. Exper. Psycho.: Gen. 136 (2007), 82-111.

[56] P. Wolff. 2012. Representing verbs with force vectors. Theor. Ling. 38 (2012), 237-248.

[57] A.-L. Mealier, G. Pointeau, P. Gärdenfors, and P. F. Dominey. 2016. Construals of meaning: The role of attention in robotic language production. Interact. Stud. 17, 1 (2016), 48-76.

[58] J. Lyons. 1977. Semantics. Cambridge University Press, Cambridge, MA.

[59] T. Regier, C. Kemp, and P. Kay. 2015. Word meanings across languages support efficient communication. In Handbook of Language Emergence, B. MacWinnhey and W. O’Grady (Eds.). Wiley, Hoboken NJ, 237-263.

[60] I. Douven and P. Gärdenfors. To appear. What are natural concepts? A design perspective. Mind Lang. DOI : 10.1111/ mila. 12240

[61] C. Paradis. 2005. Ontologies and construals in lexical semantics. Axiomathes 15 (2005) 541-573.

[62] D. Ravid. 2006. Semantic development in textual contexts during the school years: Noun scale analyses. F. Child Lang. 33 (2006) 791-821.

[63] M. Rappaport Hovav and B. Levin. 2010. Reflections on manner/result complementarity. In Lexical Semantics, Syntax, and Event Structure, M. Rappaport Hovav, D. Doron. and I. Sichel (Eds.). Oxford University Press, Oxford, UK, 21-38.

[64] L. Talmy. 2001. Toward a cognitive semantics. Typology and Process in Concept Structuring, Vol. 2. The MIT Press, Cambridge, MA.

[65] A. Cangelosi, G. Metta, G. Sagerer, S. Nolfi, C. Nehaniv, K. Fischer, T. Belpaeme, G. Sandini, F. Nori, L. Fadiga, B. Wrede, K. Rohlfing, E. Tuci, K. Dautenhahn, J. Saunders, and A. Zeschel. 2008. The iTALK project: Integration 
and transfer of action and language knowledge in robots. In Proceedings of the 3rd ACM/IEEE International Conference on Human Robot Interaction 2 (2008), 167-179.

[66] S. Kalkan, N. Dag, O. Yüruten, A. M. Borghi, and E. Sahin. 2014. Verb concepts from affordances. Interact. Stud. 15, 1 (2014), 1-37.

[67] S. Lallée, C. Madden, M. Hoen, and P. Dominey. 2010. Linking language with embodied teleological representations of action for humanoid cognition. Fron. Neurobot. DOI : 10.3389/fnbot.2010.00008

[68] Y. Demiris and B. Khadhouri. 2006. Hierarchical attentive multiple models for execution and recognition of actions. Rob. Autonom. Syst. 54 (2006), 361-369.

[69] J. Zwarts and P. Gärdenfors. 2016. Locative and directional prepositions in conceptual spaces: The role of polar convexity. F. Log., Lang. Inform. 25 (2016), 109-138.

[70] C. Vandeloise. 1986. L'espace en Français: Sémantique Des Prépositions Spatiales. Editions du Seuil, Paris.

[71] P. Gärdenfors and M. Brala. 2018. Semantic domains of demonstratives and articles: A view of deictic referentiality explored on the paradigm of Croatian demonstratives. Lingua 201 (2018), 102-118. DOI : 10.1016/j.lingua.2017.10.006

[72] H. Clark. 1996. Using Language. Cambridge University Press, Cambridge, MA.

[73] A. Cangelosi. 2010. Grounding language in action and perception: From cognitive agents to humanoid robots. Phys. Life Rev. 7 (2010), 139-151.

[74] M.-A. Williams, J. McCarthy, P. Gärdenfors, C. Stanton, and A. Karol. 2009. A grounding framework. F. Autonom. Agents Multi-Agent Sys. 19 (2009), 272-296.

[75] P. Gärdenfors. 2012. Reasons for meanings: A theory of semantics grounded in perception, action and interaction. In Welt der Gründe, J. Nida-Rümelin and E. Özmen (Eds.). Felix Meiner Verlag, Hamburg, 1327-1344.

[76] R. Pirrone and A. Chella. 2019. Grounding concepts as clusters in multiple conceptual spaces. Papers of the Towards Conscious AI Symposium co-located with the Association for the Advancement of Artificial Intelligence 2019 Spring Symposium Series (AAAI SSS-19).

[77] C. Matuszek, N. FitzGerald, L. Zettlemyer, B. Liefeng, and D. Fox. 2012. A joint model of language and perception for grounded attribute learning. Retrieved from: ArXiv Preprint Arxiv:1206.6423.

[78] C. Matuszek, E. Herbst, L. Zettlemeyer, and D. Fox. 2013. Learning to parse natural language commands to a robot control system. In Experimental Robotics. Springer, Heidelberg, 403-415.

[79] S. Tellex, P. Thker, J. Joseph, and N. Roy. 2014. Learning perceptually grounded word meaning from unaligned parallel data. Mach. Learn. 94, 2 (2014), 151-167.

[80] C. F. Hockett. 1960. The origin of speech, Sci. Amer. 203, 3 (1960), 88-96.

[81] A. Gatt and E. Krahmer. 2018. Survey of the state of art in natural language generation: Core tasks, applications and evaluation. F. Artific. Intell. Res. 61 (2018) 65-170.

[82] W. Croft and E. J. Wood. 2000. Construal operations in linguistics and artificial intelligence. In Meaning and Cognition: A Multidisciplinary Approach, L. Albertazzi (Ed.). John Benjamins, Amsterdam, 51-78.

[83] R. W. Langacker. 2008. Cognitive Grammar: A Basic Introduction. Oxford University Press, Oxford, UK.

[84] R. W. Langacker. 1987. Foundations of Cognitive Grammar, Vol. 1. Stanford University Press, Stanford, CA.

[85] T. Givón. 2001. Syntax, Vol I. John Benjamins, Philadelphia, PA.

[86] P. Dominey and J. Boucher. 2005. Learning to talk about events from narrated video in a construction grammar framework. Artific. Intell. 167 (1-2), 31-61.

[87] L. Talmy. 1985. Lexicalization patterns: Semantic structure in lexical forms. In Language Typology and Syntactic Description 3: Grammatical Categories and the Lexicon, T. Shopen (Ed.). Cambridge University Press, Cambridge, MA, 57-149.

[88] X. Hinaut and P. F. Dominey. 2014. Real-time parallel processing of grammatical structure in the fronto-striatal system: A recurrent network simulation study using reservoir computing. PLoS One 8, 2 (2014), 1-18.

[89] P. A. Morse, P. Baxter, T. Belpaeme, L. B. Smith, and A. Cangelosi. 2011. The power of words (and space). In Proceedings of the 1st foint International Conference on Developmental Learning and on Epigenetic Robotics.

[90] J. J. Kuffner and S. M. LaValle. 2000. RRT-Connect. An efficient approach to single-query path planning. Proceedings of the IEEE International Conference on Robotics and Automation. DOI : 10.1109/ROBOT.2000.844730

Received June 2018; revised May 2019; accepted June 2019 